\title{
AN ASIAN-PACIFIC PERSPECTIVE ON SOLUTIONS FOR A MORE HUMANISTIC, HOLISTIC AND INTEGRATED MODEL OF CARE
}

\author{
Peter P. Yuen \\ Correspondence: mspeter@cpce-polyu.edu.hk
}

This Special Issue selects papers from the CPCE Health Conference 2019 held at the College of Professional \& Continuing Education (CPCE) of The Hong Kong Polytechnic University on 14 January 2019. CPCE is delighted to contribute to this Special Issue of APJHM. CPCE, together with the Hong Kong College of Health Services Executives, have been working together on many fronts with the Australian College of Health Services Management, and this Special Issue is an example of another fruitful collaboration.

"Towards a More Humanistic, Holistic and Integrated Model of Care" was chosen to be the theme of this year's conference. Health care systems often tend to deliver services that are fragmented, acute-centric, highly specialized, expensive, but not necessarily in line with the values of the patient. This conference seeks to explore studies, good practices and policies from different territories in the Asia-Pacific region that could potentially facilitate the transformation of the model of care to one that is more humanistic, holistic and integrated.

Over 200 academics and health care leaders from the Asia-Pacific region attended the conference. Speakers from Hong Kong, the Chinese mainland, Japan, South Korea, Singapore, Australia, Thailand etc. presented research findings and case studies in their respective territory and explored new ways to achieve a care model that promotes patient empowerment and enhances patients' value, choice, and dignity, in the Asia-Pacific context, in which all are experiencing rapid population ageing and the resultant pressure on their health and long term care systems.

The six articles selected for this Special Issue represent a variety of approaches to tackle the problem. To facilitate better planning, the article by Hasegawa et al. presents a method to estimate the burden of major chronic diseases to demonstrate the diversity in the pattern of diseases among different regions in Japan. $\mathrm{Ng}$ et al. 's article identifies the service gap in the Hong Kong system that needs to be addressed. Benefits from the use of different health care professionals to provide better care is illustrated by Chu et al.'s case study on the role and impact of oncology pharmacists in optimizing anti-cancer treatment for cancer patients in Hong Kong. From the prevention angle, Yick et al 's study attempts to facilitate better footwear design process to prevent undesirable future foot conditions; Kwan et al.'s article presents the use of workshops to improve the functionality of geriatric footwear for older women; and Kwong et al.'s article discusses practical plans in the promotion of appropriate use of electronic devices among adolescents. In summary, this Special Issue recommends a multidisciplinary approach in tackling the problem of the health care system that could certainly benefit from more innovations and realignment.

Peter P. Yuen

Special Issue Editor and

Dean of the College of Professional \& Continuing Education, The Hong Kong Polytechnic University

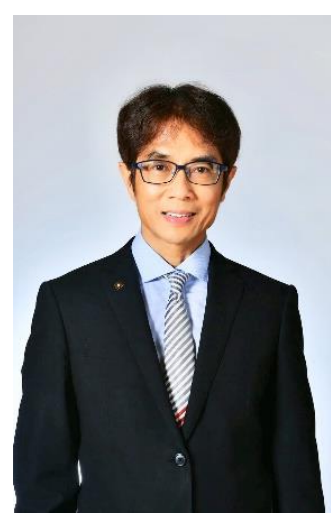

